\title{
ON A QUESTION OF KEATING AND RUDNICK ABOUT PRIMITIVE DIRICHLET CHARACTERS WITH SQUAREFREE CONDUCTOR
}

\author{
NICHOLAS M. KATZ
}

\begin{abstract}
We prove equidistribution results, in the function field setting, for the L-functions attached to primitive, odd Dirichlet characters with a fixed squarefree conductor.
\end{abstract}

\section{INTRODUCTION}

We work over a finite field $k=\mathbb{F}_{q}$ inside a fixed algebraic closure $\bar{k}$. We fix a squarefree monic polynomial $f(X) \in k[X]$ of degree $n \geq 2$. We form the $k$-algebra

$$
B:=k[X] /(f(X)),
$$

which is finite étale over $k$ of degree $n$. We denote by $u \in B$ the image of $X$ in $B$ under the "reduction mod $f$ " homomorphism $k[X] \rightarrow B$. Thus we may write this homomorphism as

$$
g(X) \in k[X] \mapsto g(u) \in B .
$$

We denote by $B^{\times}$the multiplicative group of $B$, and by $\chi$ a character

$$
\chi: B^{\times} \rightarrow \mathbb{C}^{\times} .
$$

We extend $\chi$ to all of $B$ by decreeing that $\chi(b):=0$ if $b \in B$ is not invertible.

The (possibly imprimitive) Dirichlet $L$-function $L(\chi, T)$ attached to this data is the power series in $\mathbb{C}[[T]]$ given by

$$
\begin{aligned}
L(\chi, T) & :=\sum_{\text {monic }}(\mathrm{X}(\mathrm{X}) \in \mathrm{k}[\mathrm{X}] \\
A_{n} & :=\sum_{g(X) \in k[X] \text { monic of deg. } \mathrm{n}, \operatorname{gcd}(\mathrm{f}, \mathrm{g})=1} \chi(g(u)) T^{\operatorname{deg}(g)}=\sum_{n \geq 0} A_{n} T^{n},
\end{aligned}
$$

If $\chi$ is nontrivial, then $L(\chi, T)$ is a polynomial in $T$ of degree $n-1$. 
Moreover, if $\chi$ is "as ramified as possible" ${ }^{1}$, then this L-function is "pure of weight one", i.e., in its factored form $\prod_{i=1}^{n-1}\left(1-\beta_{i} T\right)$, each reciprocal root $\beta_{i}$ has complex absolute value

$$
\left|\beta_{i}\right|_{\mathbb{C}}=\sqrt{q}
$$

For such a $\chi$, its "unitarized" $L$-function $L(\chi, T / \sqrt{q})$ is the reversed characteristic polynomial $\operatorname{det}\left(1-T A_{\chi}\right)$ of some element $A_{\chi}$ in the unitary group $U(n-1)$ (e.g., take $A_{\chi}:=\operatorname{Diag}\left(\beta_{1} / \sqrt{\mathrm{q}}, \ldots, \beta_{\mathrm{n}-1} / \sqrt{\mathrm{q}}\right)$ ). In $U(n-1)$, conjugacy classes are determined by their characteristic polynomials, so $L(\chi, T / \sqrt{q})$ is $\operatorname{det}\left(1-T \theta_{\chi}\right)$ for a well defined conjugacy class $\theta_{\chi}$ in $U(n-1)$. In order to keep track of the input data $(k, f, \chi)$, we denote this conjugacy class

$$
\theta_{k, f, \chi}
$$

Now suppose $E / k$ is a finite extension field of $k$. Our polynomial $f$ remains squarefree over $E$. We form the $E$-algebra $B_{E}:=E[X] /(f(X))$, and for each character $\chi$ of $B_{E}^{\times}$which is as ramified as possible, we get a conjugacy class $\theta_{E, f, \chi}$.

The question posed by Keating and Rudnick was to show that for fixed $f$, the collections of conjugacy classes

$$
\left\{\theta_{E, f, \chi}\right\}_{\chi} \text { char. of } \mathrm{B}_{\mathrm{E}}^{\times} \text {as ramified as possible }
$$

become equidistributed in the space $U(n-1)^{\#}$ of conjugacy classes of $U(n-1)$ (for the measure induced by Haar measure on $U(n-1))$ as $E$ runs over larger and larger finite extensions of $k$.

In fact, we will show something slightly stronger, where we fix the degree $n \geq 2$, but allow sequences of input data $\left(k_{i}, f_{i}\right)$, with $k_{i}$ a finite field (of possibly varying characteristic) and $f_{i}(X) \in k_{i}[X]$ squarefree of degree $n$. We will show that, in any such sequence in which $\# k_{i}$ is archimedeanly increasing to $\infty$, the collections of conjugacy classes

$$
\left\{\theta_{k_{i}, f_{i}, \chi}\right\}_{\chi} \text { char. of } \mathrm{B}_{\mathrm{i}}^{\times} \text {as ramified as possible }
$$

become equidistributed in $U(n-1)^{\#}$. Here is the precise statement, which occurs as Theorem 5.10 in the paper.

Theorem. Fix an integer $n \geq 2$ and a sequence of data $\left(k_{i}, f_{i}\right)$ with $k_{i}$ a finite field (of possibly varying characteristic) and $f_{i}(X) \in k_{i}[X]$

\footnotetext{
${ }^{1}$ Factor $f$ as a product of distinct monic irreducible polynomials, say $f=\prod_{j} f_{j}$. Then $B$ is canonically the product of the algebras $B_{j}:=k[X] /\left(f_{j}(X)\right)$, and $\chi$ is the product of characters $\chi_{j}$ of these factors. The condition "as ramified as possible" is that each $\chi_{j}$ be nontrivial, and that the restriction of $\chi$ to $k^{\times} \subset B^{\times}$be nontrivial. Characters satisfying this last condition, that $\chi$ be nontrivial on $k^{\times}$, are called odd.
} 
squarefree of degree $n$. If $\# k_{i}$ is archimedeanly increasing to $\infty$, the collections of conjugacy classes

$$
\left\{\theta_{k_{i}, f_{i}, \chi}\right\}_{\chi \in \operatorname{TotRam}\left(k_{i}, f_{i}\right)}
$$

become equidistributed in $U(n-1)^{\#}$ as $\# k_{i} \rightarrow \infty$.

Keating and Rudnick use this result to prove their Theorem 2.2 in [K-R], cf. [K-R, (5.16)].

In an appendix, we give an analogous result, Theorem 6.4 , for "even" characters which are as ramified as possible, given that they are even, under the additional hypothesis that each $f_{i}(X) \in k_{i}[X]$ have a zero in $k_{i}$. Here the equidistribution is in the space of conjugacy classes of $U(n-2)$. This additional hypothesis, that each $f_{i}(X) \in k_{i}[X]$ have a zero in $k_{i}$, should not be necessary, but at present we do not know how to remove it. Already the case when each $f_{i}(X) \in k_{i}[X]$ is an irreducible cubic seems to be open.

\section{Preliminaries on the $L$-FunCtion}

We return to our initial situation, a finite field $k$, an integer $n \geq 2$, a squarefree polynomial $f(X) \in k[X]$, and the finite étale $k$-algebra $B:=k[X] /(f(X))$. We have the algebra-valued functor $\mathbb{B}$ on variable $k$-algebras $R$ defined by

$$
\mathbb{B}(R):=B_{R}:=B \otimes_{k} R=R[X] /(f(X)),
$$

and the group-valued functor $\mathbb{B}^{\times}$on variable $k$-algebras $R$ defined by

$$
\mathbb{B}^{\times}(R):=B_{R}^{\times}=\mathbb{B}(R)^{\times} .
$$

Because $f$ is squarefree, $\mathbb{B}^{\times}$is a smooth commutative groupscheme ${ }^{2}$ over $k$, which over any extension field $E$ of $k$ in which $f$ factors completely becomes isomorphic to the $n$-fold product of $\mathbb{G}_{m}$ with itself. More precisely, if $f$ factors completely over $E$, say $f(X)=\prod_{i=1}^{n}(X-$ $\left.a_{i}\right)$, then for any $E$-algebra $R$, we have an $R$-algebra isomorphism

$$
\mathbb{B}(R)=R[X] /\left(\prod_{i=1}^{n}\left(X-a_{i}\right)\right) \cong \prod_{i=1}^{n} R
$$

of $\mathbb{B}(R)$ with the $n$-fold product of $R$ with itself, its algebra structure given by componentwise operations, under which the image $u$ of $X$ maps by

$$
u \mapsto\left(a_{1}, \ldots, a_{n}\right) .
$$

So for any $E$-algebra $R$, we have $\mathbb{B}^{\times}(R):=\mathbb{B}(R)^{\times} \cong\left(R^{\times}\right)^{n}$.

\footnotetext{
${ }^{2}$ In fact $\mathbb{B}^{\times}$is the generalized Jacobian of $\mathbb{P}^{1} / k$ with respect to the modulus $\infty \cup\left\{f=0\right.$ in $\left.\mathbb{A}^{1}\right\}$.
} 
For $E / k$ a finite extension field, $B_{E}$ is a finite etale $B$ algebra which as a $B$-module is free of rank $\operatorname{deg}(E / k)$. Let us denote by $\mathbb{B}_{E}$ the functor on $k$-algebras $R \mapsto \mathbb{B}_{E}(R):=B_{E} \otimes_{k} R$. Then $\mathbb{B}_{E}(R)$ is a finite étale $\mathbb{B}(R)$-algebra, so we have the norm map

$$
\operatorname{Norm}_{E / k}: \mathbb{B}_{E} \rightarrow \mathbb{B} \text {. }
$$

Its restriction to unit groups gives a homomorphism of tori which is étale surjective,

$$
\operatorname{Norm}_{E / k}: \mathbb{B}_{E}^{\times} \rightarrow \mathbb{B}^{\times}
$$

whose restriction to $k$-valued points gives a surjective ${ }^{3}$ homomorphism

$$
\operatorname{Norm}_{E / k}: \mathbb{B}^{\times}(E) \rightarrow \mathbb{B}^{\times}(k) .
$$

We will also have occasion to consider $\mathbb{B}(R)$ as a finite étale $R$-algebra which is free of rank $n$ as an $R$-module, giving us another norm map

$$
\operatorname{Norm}_{B / k}: \mathbb{B}(R) \rightarrow R,
$$

which by restriction gives a homomorphism which is étale surjective, with geometrically connected kernel,

$$
\operatorname{Norm}_{B / k}: \mathbb{B}^{\times}(R) \rightarrow R^{\times} \text {. }
$$

For any finite extension $E / k$, this second norm map

$$
\operatorname{Norm}_{B / k}: \mathbb{B}^{\times}(E) \rightarrow E^{\times}
$$

is surjective.

How is all this related to our $L$-function? For each integer $r \geq 1$, denote by $k_{r} / k$ the unique extension field of $k$ of degree $r$ (inside our fixed algebraic closure of $k$ ). Recall that $f(X) \in k[X]$ is squarefree of degree $n \geq 1$, and that $u$ denotes the image of $X$ in $B=k[X] /(f(X))$.

Lemma 1.1. For $\chi$ a character of $B$, we have the identity

$$
L(\chi, T)=\exp \left(\sum_{r \geq 1} S_{r} T^{r} / r\right), \quad S_{r}=\sum_{t \in \mathbb{A}^{1}[1 / f]\left(k_{r}\right)} \chi\left(\operatorname{Norm}_{k_{r} / k}(u-t)\right) .
$$

Proof. The key observation is that if $\alpha \in \mathbb{A}^{1}[1 / f]\left(k_{d}\right)$ generates the extension $k_{d} / k$, and has monic irreducible polynomial $P(X)$ over $k$, then $\operatorname{gcd}(f, P)=1$ and $P(X)=\operatorname{Norm}_{k_{d} / k}(X-\alpha)$ in $k[X]$. Hence $P(u)=\operatorname{Norm}_{k_{d} / k}(u-\alpha)$ in $B$. We apply this as follows.

Write the $L$-function as the Euler product

$$
L(\chi, T)=\prod_{\text {monic irred. } \mathrm{P}(\mathrm{X}), \operatorname{gcd}(\mathrm{f}, \mathrm{P})=1} \frac{1}{1-\chi(P(u)) T^{\operatorname{deg}(P)}} .
$$

\footnotetext{
${ }^{3}$ By Lang's theorem [La, Thm. 2], because its kernel is smooth and geometrically connected.
} 
Taking log's, we must check that for each $r \geq 1$ we have the identity

$$
\sum_{t \in \mathbb{A}^{1}[1 / f]\left(k_{r}\right)} \chi\left(\operatorname{Norm}_{k_{r} / k}(u-t)\right)=\sum_{d \mid r} \sum_{\text {irred } \mathrm{P}, \operatorname{deg}(\mathrm{P})=\mathrm{d}, \operatorname{gcd}(\mathrm{f}, \mathrm{P})=1} d \chi(P(u))^{r / d} .
$$

To see this, partition the points $t \in \mathbb{A}^{1}[1 / f]\left(k_{r}\right)$ according to their monic irreducible polynomials over $k$. For each divisor $d$ of $r$, and each monic irreducible $P(X)$ of degree $d$ with $\operatorname{gcd}(f, P)=1$ and roots $\tau_{1}, \ldots, \tau_{d}$ in $\mathbb{A}^{1}[1 / f]\left(k_{d}\right)$, each of the $d$ terms $\chi\left(\operatorname{Norm}_{k_{r} / k}\left(u-\tau_{i}\right)\right)$ is equal to $\chi(P(u))^{r / d}$ (simply because $\operatorname{Norm}_{k_{d} / k}\left(u-\tau_{i}\right)=P(u)$, and, as $\tau_{i} \in k_{d}$, $\left.\operatorname{Norm}_{k_{r} / k}\left(u-\tau_{i}\right)=\left(\operatorname{Norm}_{k_{d} / k}\left(u-\tau_{i}\right)\right)^{r / d}\right)$.

\section{Cohomological genesis}

We now choose a prime number $\ell$ invertible in $k$, and an embedding of $\overline{\mathbb{Q}}$, the algebraic closure of $\mathbb{Q}$ in $\mathbb{C}$, into $\overline{\mathbb{Q}}$. In this way, we view $\chi$ as a $\overline{\mathbb{Q}}_{\ell} \times$-valued character of $B^{\times}$. Attached to $\chi$, we have the "Kummer sheaf" $\mathcal{L}_{\chi}$ on $\mathbb{B}^{\times}$. Recall that $\mathcal{L}_{\chi}$ is obtained as follows. We have the $q=\# k^{\prime}$ 'th power Frobenius endomorphism $F_{k}$ of $\mathbb{B}$. The Lang torsor, i.e., the finite étale galois covering $1-F_{k}: \mathbb{B}^{\times} \rightarrow \mathbb{B}^{\times}$, has structural group $B^{\times}=\mathbb{B}^{\times}(k)$. We then push out this $B^{\times}$-torsor on $\mathbb{B}^{\times}$by $\bar{\chi}$, to obtain the $\overline{\mathbb{Q}_{\ell}}$-sheaf $\mathcal{L}_{\chi}$ on $\mathbb{B}^{\times}$. It is lisse of rank one and pure of weight zero.

We have a $k$-morphism (in fact an embedding)

$$
\mathbb{A}^{1}[1 / f] \subset \mathbb{B}^{\times},
$$

given on $R$-valued points, $R$ any $k$-algebra, by

$$
t \in \mathbb{A}^{1}[1 / f](R) \mapsto u-t \in \mathbb{B}(R) .
$$

Lemma 2.1. For any $k$-algebra $R$, and any $t \in \mathbb{A}^{1}(R)=R$, we have the identity

$$
\operatorname{Norm}_{B / k}(u-t)=(-1)^{n} f(t) \in R .
$$

Proof. In the $k$-algebra $B=k[X] /(f(X))$, multiplication by $u$ (the class of $X$ in $B$ ) has characteristic polynomial $f$ (theory of the "companion matrix"), i.e., taking for $R$ the polynomial ring $k[T]$, we have $\operatorname{Norm}_{B / k}(T-u)=f(T) \in R=k[T]$, hence $\operatorname{Norm}_{B / k}(u-T)=$ $(-1)^{n} f(T) \in k[T]$, and this is the universal case of the asserted identity.

We denote by $\mathcal{L}_{\chi(u-t)}$ the lisse $\overline{\mathbb{Q}_{\ell}}$-sheaf of rank one on $\mathbb{A}^{1}[1 / f]$ obtained as the pullback of $\mathcal{L}_{\chi}$ on $\mathbb{B}^{\times}$by the embedding $t \mapsto u-t$ of $\mathbb{A}^{1}[1 / f]$ into $\mathbb{B}^{\times}$. In view of Lemma 1.1 , the $L$-function $L(\chi, T)$ is, via the chosen embedding of $\overline{\mathbb{Q}}$ into $\overline{\mathbb{Q}}$, the $L$-function of $\mathbb{A}^{1}[1 / f] / k$ with 
coefficients in $\mathcal{L}_{\chi(u-t)}$. This sheaf on $\mathbb{A}^{1}[1 / f]$ is lisse of rank one and pure of weight zero. The compact cohomology groups

$$
H_{c}^{i}:=H_{c}^{i}\left(\mathbb{A}^{1}[1 / f] \otimes_{k} \bar{k}, \mathcal{L}_{\chi(u-t)}\right)
$$

vanish for $i \neq 1,2$, and by the Lefschetz trace formula we have the formula

$$
L(\chi, T)=\operatorname{det}\left(1-\text { TFrob }_{k} \mid H_{c}^{1}\right) / \operatorname{det}\left(1-\text { TFrob }_{k} \mid H_{c}^{2}\right) .
$$

We now turn to a closer examination of these cohomology groups. For this, we first examine the sheaf $\mathcal{L}_{\chi(u-t)}$ geometrically, i.e., pulled back to $\mathbb{A}^{1}[1 / f] / \bar{k}$, and describe it in terms of translations of Kummer sheaves $\mathcal{L}_{\rho}$ on $\mathbb{G}_{m}$. Recall that the tame fundamental group $\pi_{1}^{\text {tame }}\left(\mathbb{G}_{m} / \bar{k}\right)$ is the inverse limit over prime-to $p$ integers $N$, growing multiplicatively, of the groups $\mu_{N}(\bar{k})$, via the $N$ 'th power Kummer coverings of $\mathbb{G}_{m} / \bar{k}$ by itself. It is also the inverse limit, over finite extension fields $E / k$ growing by inclusion, of the multiplicative groups $E^{\times}$, with transition maps the Norm, via the Lang torsor coverings $1-F_{E}$ of of $\mathbb{G}_{m} / \bar{k}$ by itself. For any continuous $\overline{\mathbb{Q}}_{\ell}{ }^{x}$-valued character $\rho$ of $\pi_{1}^{\text {tame }}\left(\mathbb{G}_{m} / \bar{k}\right)$, we have the corresponding Kummer sheaf $\mathcal{L}_{\rho}$ on $\mathbb{G}_{m} / \bar{k}$. The characters of finite order of $\pi_{1}^{\text {tame }}\left(\mathbb{G}_{m} / \bar{k}\right)$ are precisely those which arise from characters $\rho$ of $E^{\times}$for some finite extension $E / k$. More precisely, a character $\rho$ of finite order of $\pi_{1}^{\text {tame }}\left(\mathbb{G}_{m} / \bar{k}\right)$ comes from a character of $E^{\times}$if and only if $\rho=\rho^{\# E}$ (equality as characters of $\pi_{1}^{\text {tame }}\left(\mathbb{G}_{m} / \bar{k}\right)$ ). For such a character $\rho$, the Kummer sheaf $\mathcal{L}_{\rho}$ on $\mathbb{G}_{m} / \bar{k}$ begins life on $\mathbb{G}_{m} / E$.

To analyze the sheaf $\mathcal{L}_{\chi(u-t)}$ geometrically, first choose a finite extension field $E / k$ in which $f$ factors completely, say $f(X)=\prod_{i=1}^{n}\left(X-a_{i}\right)$. Then $\mathbb{B}(E)^{\times} \cong\left(E^{\times}\right)^{n}$, and $\chi_{E}:=\chi \circ \operatorname{Norm}_{E / k}$ as character of $\left(E^{\times}\right)^{n}$ is of the form $\left(x_{1}, \ldots, x_{n}\right) \mapsto \prod \chi_{i}\left(x_{i}\right)$, for characters $\chi_{1}, \ldots, \chi_{n}$ of $E^{\times}$. Then $\mathbb{B}^{\times}$, pulled back to $\bar{k}$, becomes $\mathbb{G}_{m}^{n}$, and $\mathcal{L}_{\chi}$ on it becomes the external tensor product $\bigotimes_{i=1}^{n} \mathcal{L}_{\chi_{i}}$ of usual Kummer sheaves $\mathcal{L}_{\chi_{i}}$ on the factors. Over $\bar{k}$, the embedding of $\mathbb{A}^{1}[1 / f]$ into $\mathbb{B}^{\times}$given by $t \mapsto u-t$ becomes the embedding of $\mathbb{A}^{1}[1 / f] \otimes_{k} \bar{k}$ into $\mathbb{G}_{m}^{n}$ given by

$$
t \mapsto\left(a_{1}-t, \ldots, a_{n}-t\right) .
$$

Thus the sheaf $\mathcal{L}_{\chi(u-t)}$ is geometrically isomorphic to the tensor product $\otimes_{i=1}^{n} \mathcal{L}_{\chi_{i}\left(a_{i}-t\right)}$ on $\mathbb{A}^{1}[1 / f] \otimes_{k} \bar{k}=\operatorname{Spec}(\bar{k}[t][1 / f(t)])$.

Lemma 2.2. With the notations of the previous paragraph, we have the following results.

(1) We have $H_{c}^{2}=0$ if and only if some $\chi_{i}$ is nontrivial, in which case $H_{c}^{1}$ has dimension $n-1$. 
(2) The group $H_{c}^{1}$ is pure of weight one if and only if every $\chi_{i}$ is nontrivial and the product $\prod_{i=1}^{n} \chi_{i}$ is nontrivial.

Proof. Both assertions are invariant under finite extension of the ground field, so it suffices to treat universally the case in which $f$ factors completely over $k$. The character $\chi_{i}$ is the local monodromy of $\mathcal{L}_{\chi(u-t)}$ at the point $a_{i}$, and the product $\prod_{i=1}^{n} \chi_{i}$ is its local monodromy at $\infty$. For assertion (1), we note that the group $H_{c}^{2}$ is either zero or onedimensional. It is nonzero if and only if the lisse rank one sheaf $\mathcal{L}_{\chi(u-t)}$ is geometrically constant, i.e., if and only if its local monodromy at each of the points $\infty, a_{1}, \ldots, a_{n}$ is trivial. The dimension assertion results from the Euler-Poincaré formula: because $\mathcal{L}_{\chi(u-t)}$ is lisse of rank one and at worst tamely ramified at the missing points, it gives

$$
\chi_{c}\left(\mathbb{A}^{1}[1 / f] \otimes_{k} \bar{k}, \mathcal{L}_{\chi(u-t)}\right)=\chi_{c}\left(\mathbb{A}^{1}[1 / f] \otimes_{k} \bar{k}, \overline{\mathbb{Q}_{\ell}}\right)=1-n .
$$

For assertion (2), we argue as follows. If all the $\chi_{i}$ are trivial, i.e., if $\chi$ is trivial, then $\mathcal{L}_{\chi}$ on $\mathbb{B}^{\times}$is trivial, $\mathcal{L}_{\chi(u-t)}$ on $\mathbb{A}^{1}[1 / f]$ is trivial, and its $H_{c}^{1}$ has dimension $n$ and is pure of weight zero.

Suppose now that $\chi$ is nontrivial, i.e., that at least one $\chi_{i}$ is nontrivial. Denote by $j: \mathbb{A}^{1}[1 / f] \subset \mathbb{P}^{1}$ the inclusion. Then we have a short exact sequence of sheaves on $\mathbb{P}^{1}$

$$
0 \rightarrow j_{!} \mathcal{L}_{\chi(u-t)} \rightarrow j_{\star} \mathcal{L}_{\chi(u-t)} \rightarrow P c t \rightarrow 0,
$$

in which Pct is a skyscraper sheaf, supported at those of the points $\infty, a_{1}, \ldots, a_{n}$ where the local monodromy is trivial, and is punctually pure of weight zero with one-dimensional stalk at each of these points. The long exact cohomology sequence then gives a short exact sequence

$$
0 \rightarrow H^{0}\left(\mathbb{P}^{1} / \bar{k}, P c t\right) \rightarrow H^{1}\left(\mathbb{P}^{1} / \bar{k}, j_{!} \mathcal{L}_{\chi(u-t)}\right) \rightarrow H^{1}\left(\mathbb{P}^{1} / \bar{k}, j_{\star} \mathcal{L}_{\chi(u-t)}\right) \rightarrow 0
$$

in which the middle term $H^{1}\left(\mathbb{P}^{1} / \bar{k}, j_{!} \mathcal{L}_{\chi(u-t)}\right)$ is the cohomology group $H_{c}^{1}$, the third term $H^{1}\left(\mathbb{P}^{1} / \bar{k}, j_{\star} \mathcal{L}_{\chi(u-t)}\right)$ is pure of weight one [De-Weil II, 3.2.3], and the first term, $H^{0}\left(\mathbb{P}^{1} / \bar{k}, P c t\right)$ is pure of weight zero and of dimension the number of points among $\infty, a_{1}, \ldots, a_{n}$ where the local monodromy is trivial.

Given a character $\chi$ of $B^{\times}$, how do we determine what $\mathcal{L}_{\chi(u-t)}$ looks like, geometrically? We know that, in terms of the factorization of $f$, say $f(X)=\prod_{i=1}^{n}\left(X-a_{i}\right)$ over some finite extension field $E / k$, $\mathcal{L}_{\chi(u-t)}$ is geometrically isomorphic to the tensor product $\otimes_{i=1}^{n} \mathcal{L}_{\chi_{i}\left(a_{i}-t\right)}$ on $\mathbb{A}^{1}[1 / f] \otimes_{k} \bar{k}=\operatorname{Spec}(\bar{k}[t][1 / f(t)])$. We have an easy interpretation of the product $\prod_{i} \chi_{i}$ of all the $\chi_{i}$. 
Lemma 2.3. For $\rho:=$ the restriction of $\chi$ to $k^{\times}\left(k^{\times}\right.$seen as a subgroup of $\left.B^{\times}\right)$, the composition $\rho \circ \operatorname{Norm}_{E / k}$ is the character of $E^{\times}$given by the product $\prod_{i} \chi_{i}$ of all the $\chi_{i}$.

Proof. Under the $E$-linear isomorphism of $B_{E}=E[X] /(f)$ with the $n$-fold self product of $E, E$ viewed as the constant polynomials is diagonally embedded. Thus $\prod_{i} \chi_{i}$ is the effect of $\chi \circ \operatorname{Norm}_{B_{E} / B}$ on $E^{\times}$ (viewed as a subgroup of $B_{E}^{\times}$). The restriction to $E^{\times}$of this norm map $\operatorname{Norm}_{B_{E} / B}: B_{E}^{\times} \rightarrow B^{\times}$is the norm map $\operatorname{Norm}_{E / k}: E^{\times} \rightarrow k^{\times}$.

To further analyze this question, in a " $k$-rational" way, we first factor our squarefree monic $f$ as a product of distinct monic $k$-irreducible polynomials, say

$$
f=\prod P_{i}, \operatorname{deg}\left(P_{i}\right):=d_{i}
$$

Then with

$$
B_{P_{i}}:=k[X] /\left(P_{i}\right),
$$

we have an isomorphism of $k$-algebras

$$
B:=k[X] /(f) \cong \prod_{i} B_{P_{i}}, g \mapsto\left(g \bmod P_{i}\right)_{i},
$$

and a character $\chi$ of $B^{\times}$is uniquely of the form

$$
\chi(g)=\prod_{i} \chi_{P_{i}}\left(g \bmod P_{i}\right),
$$

for characters $\chi_{P_{i}}$ of $B_{P_{i}}^{\times}$.

So it suffices treat the case when $f$ is a single irreducible polynomial $P$ of some degree $d \geq 1$. Choose a root $a$ of $P$ in our chosen $\bar{k}$. This choice gives an isomorphism of $B_{P}$ with the unique extension field $k_{d} / k$ of degree $d_{i}$ inside $\bar{k}$, namely $g \mapsto g(a)$. Via this isomorphism, the character $\chi_{P}$ becomes a character $\chi$ of $k_{d}^{\times}$. After extension of scalars from $k$ to $k_{d}$, we have a $k_{d}$-linear isomorphism

$$
B_{P} \otimes_{k} k_{d}=k_{d}[X] /(P) \cong \prod_{\sigma \in \operatorname{Gal}\left(k_{d} / k\right)} k_{d}, g(X) \mapsto\left(g(\sigma(a))_{\sigma}\right.
$$

Then for $g(X) \in k_{d}[X] /\left(P_{i}\right)$, its $k_{d} / k$-Norm down to $B_{P}$ is

$$
\operatorname{Norm}_{k_{d} / k}(g(X))=\prod_{\tau \in \operatorname{Gal}\left(k_{d} / k\right)} g^{\tau}(X) \bmod P=\prod_{\tau \in \operatorname{Gal}\left(k_{d} / k\right)} g^{\tau}(a) \in k_{d} \text {. }
$$

So we have the identity

$$
\left(\chi \circ \operatorname{Norm}_{k_{d} / k}\right)(g(X))=\prod_{\tau \in \operatorname{Gal}\left(k_{d} / k\right)} \chi\left(g^{\tau}(a)\right)=\prod_{\tau \in \operatorname{Gal}\left(k_{d} / k\right)}(\chi \circ \tau)\left(g\left(\tau^{-1}(a)\right) .\right.
$$


The arguments $g\left(\tau^{-1}(a)\right)$ of the characters $\chi \circ \tau$ are just the components, in another order, of $g$ in the isomorphism $k_{d}[X] /(P) \cong \prod_{\sigma \in \operatorname{Gal}\left(k_{d} / k\right)} k_{d}$. In other words, the pullback of $\chi$ by the $k_{d} / k$-Norm from $B_{P} \otimes_{k} k_{d}$ down to $B_{P}$ has components $\left(\chi, \chi^{q}, \ldots, \chi^{q^{d-1}}\right)$. Thus we have the following lemma.

Lemma 2.4. For $P$ an irreducible monic $k$-polynomial of degree $d \geq 1$, and $\chi$ a character of $B_{P}^{\times} \cong k_{d}^{\times}$(via $u \mapsto a$, a a chosen root of $P$ in $\left.k_{d}\right)$, the sheaf $\mathcal{L}_{\chi}(u-t)$ on $\mathbb{A}^{1}[1 / P]$ is geometrically isomorphic to the tensor product $\otimes_{i=0}^{d-1} \mathcal{L}_{\chi^{q^{i}}\left(a^{q^{-i}}-t\right)}$.

Combining these last two lemmas with Lemma 2.2, we get the following result.

Lemma 2.5. Let $f$ be a squarefree monic $k$-polynomial of degree $n \geq 2$, $f=\prod_{i} P_{i}$ its factorization into monic $k$-irreducibles, $\chi$ a character of $B^{\times}$, and, for each $P_{i}, \chi_{P_{i}}$ the $P_{i}$-component of $\chi$. The group $H_{c}^{1}\left(\mathbb{A}^{1}[1 / f] \otimes_{k} \bar{k}, \mathcal{L}_{\chi(u-t)}\right)$ is pure of weight one if and only if $\chi$ is nontrivial on $k^{\times}$and each $\chi_{P_{i}}$ is nontrivial, in which case $H_{c}^{1}$ has dimension $n-1$ and $H_{c}^{2}=0$.

\section{The DIRECT IMAGE THEOREM}

In this section, we work over $\bar{k}$. The following theorem gives sufficient ${ }^{4}$ conditions for a certain perverse sheaf to be irreducible (part (2)) and in addition to be isomorphic to no nontrivial multiplicative translate of itself (part (3)). This result will allow us, in sections 4 and 5 , to apply the theory developed in [Ka-CE].

Theorem 3.1. Suppose that $f(X)=\prod_{i=1}^{n}\left(X-a_{i}\right)$ is a squarefree polynomial of degree $n \geq 2$ over $\bar{k}$. Let $\chi_{1}, \ldots, \chi_{n}$ be characters of $\pi_{1}^{\text {tame }}\left(\mathbb{G}_{m} / \bar{k}\right)$ of finite order, and form the lisse sheaf

$$
\mathcal{F}:=\otimes_{i=1}^{n} \mathcal{L}_{\chi_{i}\left(a_{i}-t\right)}
$$

on $\mathbb{A}^{1}[1 / f] \otimes_{k} \bar{k}$. Then we have the following results.

(1) For any scalar $\lambda \in \bar{k}^{\times}$, the direct image $[\lambda f]_{\star} \mathcal{F}$ of $\mathcal{F}$ by the polynomial map $\lambda f: \mathbb{A}^{1}[1 / f] / \bar{k} \rightarrow \mathbb{G}_{m} / \bar{k}$ is a middle extension sheaf on $\mathbb{G}_{m} / \bar{k}$, of generic rank $n$, and the perverse sheaf $[\lambda f]_{\star} \mathcal{F}[1]$ is geometrically semisimple.

(2) If one of the $\chi_{i}$, say $\chi_{1}$, is a singleton among the $\chi^{\prime}$ 's, in the sense that $\chi_{1} \neq \chi_{j}$ for every $j \neq 1$, then the perverse sheaf $[\lambda f]_{\star} \mathcal{F}[1]$ on on $\mathbb{G}_{m} / \bar{k}$ is irreducible.

\footnotetext{
${ }^{4}$ There is no reason to think that this result is optimal.
} 
(3) If two of the $\chi_{i}$, say $\chi_{1}$ and $\chi_{2}$ are each singletons among the $\chi$ 's, then the irreducible perverse sheaf $[\lambda f]_{\star} \mathcal{F}[1]$ on on $\mathbb{G}_{m} / \bar{k}$ is not isomorphic to any nontrivial multiplicative translate of itself.

Proof. To prove (1), we argue as follows. The map $\lambda f: \mathbb{A}^{1}[1 / f] / \bar{k} \rightarrow$ $\mathbb{G}_{m} / \bar{k}$ is finite and flat of degree $n$. As $f$ has all distinct roots, its derivative $f^{\prime}$ is not identically zero, so over the dense open set $U$ of $\mathbb{G}_{m} / \bar{k}$ obtained by deleting the images under $\lambda f$ of the zeroes of $f^{\prime}$, the map $\lambda f$ is finite étale of degree $n$. Thus $[\lambda f]_{\star} \mathcal{F}[1]$ has generic rank $n$. It is a middle extension because $\mathcal{F}$ is a middle extension on the source (being lisse), and $\lambda f$ is finite, flat, and generically étale, cf. [Ka-TLFM, first paragraph of the proof of 3.3.1]. On the dense open set $U,[\lambda f]_{\star} \mathcal{F}$ is (the pullback from some finite subfield $E$ of $\bar{k}$ of) a lisse sheaf which is pure of weight zero, hence is geometrically semisimple [De-Weil II, 3.4 .1 (iii)]. Therefore [BBD, 4.3.1 (ii)] the perverse sheaf $[\lambda f]_{\star} \mathcal{F}[1] \mid U$ is semisimple, and this property is preserved by middle extension from $U$ to $\mathbb{G}_{m} / \bar{k}$.

Suppose now that $\chi_{1}$ is a singleton among the $\chi^{\prime}$ 's. We claim that $[\lambda f]_{\star} \mathcal{F}[1]$ is irreducible. Since $[\lambda f]_{\star} \mathcal{F}[1]$ is just a multiplicative translate of $f_{\star} \mathcal{F}[1]$, it suffices to show that $f_{\star} \mathcal{F}[1]$ is irreducible. Since $f_{\star} \mathcal{F}[1]$ is semisimple, we must show that the inner product

$$
<f_{\star} \mathcal{F}[1], f_{\star} \mathcal{F}[1]>=1 .
$$

By Frobenius reciprocity, we have

$$
<f_{\star} \mathcal{F}[1], f_{\star} \mathcal{F}[1]>=<\mathcal{F}[1], f^{\star} f_{\star} \mathcal{F}[1]>.
$$

So we must show that $\mathcal{F}[1]$ occurs at most once in $f^{\star} f_{\star} \mathcal{F}[1]$. We will show the stronger statement, that denoting by $I\left(a_{1}\right)$ the inertia group at the point $a_{1} \in \mathbb{A}^{1}(\bar{k})$, the $I\left(a_{1}\right)$-representation of $\mathcal{F}[1]$ occurs at most once in the $I\left(a_{1}\right)$-representation of $f^{\star} f_{\star} \mathcal{F}[1]$. As a finite flat map of $\mathbb{A}^{1}$ to itself, $f$ is finite étale over a neighborhood of 0 in the target (because $f$ has $n$ distinct roots $a_{1}, \ldots, a_{n}$, the preimages of 0$)$. We first infer that the $I(0)$-representation of $f_{\star} \mathcal{F}[1]$ is the direct sum of the $\chi_{i}$, and then that for each $j$ the $I\left(a_{j}\right)$-representation of $f^{\star} f_{\star} \mathcal{F}[1]$ is the direct sum of the $\chi_{i}$. At the point $a_{1}$, the $I\left(a_{1}\right)$-representation of $\mathcal{F}[1]$ is $\chi_{1}$, and by the singleton hypothesis $\chi_{1}$ occurs only once in the direct sum of the $\chi_{i}$, so only once in the $I\left(a_{1}\right)$-representation of $f^{\star} f_{\star} \mathcal{F}[1]$.

Suppose now that both $\chi_{1}$ and $\chi_{2}$ are singletons. We must show that for any scalar $\lambda \neq 1$ in $\bar{k}^{\times}$, the perverse irreducible sheaves $[\lambda f]_{\star} \mathcal{F}[1]$ and $f_{\star} \mathcal{F}[1]$ on $\mathbb{G}_{m} / \bar{k}$ are not isomorphic. We argue by contradiction, and thus suppose the two are isomorphic. Choose a finite subfield $E$ of $\bar{k}$ 
over which the scalar $\lambda$, the points $a_{i}$, the characters $\chi_{i}$ and the open set $U$ are all defined, so that we may speak of the geometrically irreducible perverse sheaves $[\lambda f]_{\star} \mathcal{F}(1 / 2)[1]$ and $f_{\star} \mathcal{F}(1 / 2)[1]$ on $\mathbb{G}_{m} / E$. Each of these is pure of weight zero. On the dense open set $U \subset \mathbb{G}_{m} / E$, the sheaves $[\lambda f]_{\star} \mathcal{F}$ and $f_{\star} \mathcal{F}$ are lisse and geometrically isomorphic, so one is a constant field twist of the other, say $[\lambda f]_{\star} \mathcal{F}\left|U \cong f_{\star} \mathcal{F} \otimes \alpha^{\operatorname{deg}}\right| U$, for some scalar $\alpha \in \overline{\mathbb{Q}}_{\ell} \times$. Taking middle extensions, we find an arithmetic isomorphism

$$
[\lambda f]_{\star} \mathcal{F}(1 / 2)[1] \cong f_{\star} \mathcal{F}(1 / 2)[1] \otimes \alpha^{\operatorname{deg}}
$$

on $\mathbb{G}_{m} / E$. Because both $[\lambda f]_{\star} \mathcal{F}(1 / 2)[1]$ and $f_{\star} \mathcal{F}(1 / 2)[1]$ are pure of weight zero, the scalar $\alpha$ must be pure of weigh zero. This arithmetic isomorphism implies that (and, given the geometric irreducibility, is in fact equivalent to the fact that) for any finite extension $L / E$, and any point $t \in L^{\times}$, we have an equality of traces

$$
\operatorname{Trace}\left(F r o b_{L, t} \mid[\lambda f]_{\star} \mathcal{F}(1 / 2)\right)=\alpha^{\operatorname{deg}(L / E)} \operatorname{Trace}\left(F_{\operatorname{rob}}{ }_{L, t} \mid f_{\star} \mathcal{F}(1 / 2)\right) .
$$

Because $\left.[\lambda f]_{\star} \mathcal{F}(1 / 2)[1]\right)$ is a geometrically irreducible perverse sheaf on $\mathbb{G}_{m} / E$ which is pure of weight zero, we have the estimate, as $L / E$ runs over larger and larger finite extensions,

$$
\sum_{t \in \mathbb{G}_{m}(L)} \mid \operatorname{Trace}\left(\operatorname{Frob}_{L, t}\left|[\lambda f]_{\star} \mathcal{F}(1 / 2)\right|^{2}=1+O(1 / \sqrt{\# L}),\right.
$$

or equivalently the estimate

$$
\sum_{t \in \mathbb{G}_{m}(L)} \mid \operatorname{Trace}\left(\operatorname{Frob}_{L, t}\left|[\lambda f]_{\star} \mathcal{F}\right|^{2}=\# L+O(\sqrt{\# L})\right.
$$

Indeed, it suffices to check that this second estimate holds instead for the sum over points $t \in U(L)$, as this sum omits at most \# $\left(\mathbb{G}_{m} \backslash U\right)(\bar{k})$ terms, each of which is itself $O(1)$. Because $[\lambda f]_{\star} \mathcal{F}$ is lisse on $U$ and pure of weight zero, the sum over $U$ is given, by the Lefschetz trace formula, in terms of the sheaf $\operatorname{End}:=\operatorname{End}\left([\lambda f]_{\star} \mathcal{F}\right)$ as

$$
\operatorname{Trace}\left(F r o b_{L} \mid H_{c}^{2}(U / \bar{k}, E n d)-\operatorname{Trace}\left(F r o b_{L} \mid H_{c}^{1}(U / \bar{k}, E n d) .\right.\right.
$$

The sheaf End is pure of weight zero. By the geometric irreducibility of $\left([\lambda f]_{\star} \mathcal{F}\right) \mid U$, the $\pi_{1}^{\text {geom }}(U)$-coinvariants of End are just the constants $\overline{\mathbb{Q}_{\ell}}$, so the group $H_{c}^{2}$ above is just $\overline{\mathbb{Q}_{\ell}}(-1)$, on which $F_{r o b}$ acts as $\# L$. The $H_{c}^{1}$ group is mixed of weight $\leq 1$, so we get the asserted estimate.

We now rewrite the sum of squares as follows. The sheaves $\mathcal{F}$ and

$$
\overline{\mathcal{F}}:=\otimes_{i=1}^{n} \mathcal{L}_{\overline{\chi_{i}}}\left(a_{i}-t\right)
$$


have complex conjugate trace functions, as do the their direct images by any $\lambda f$. As $\alpha$ is pure of weight zero, we have $\bar{\alpha}=1 / \alpha$. So we have

$$
\begin{gathered}
\alpha^{\operatorname{deg}(L / E)} \sum_{t \in \mathbb{G}_{m}(L)} \mid \operatorname{Trace}\left(\operatorname{Frob}_{L, t}\left|[\lambda f]_{\star} \mathcal{F}\right|^{2}=\right. \\
=\sum_{t \in \mathbb{G}_{m}(L)}\left(\operatorname{Trace}\left(\operatorname{Frob}_{L, t} \mid[\lambda f]_{\star} \mathcal{F}\right)\right)\left(\operatorname{Trace}\left(\operatorname{Frob}_{L, t} f_{\star} \overline{\mathcal{F}}\right)\right)= \\
=\alpha^{\operatorname{deg}(L / E)} \# L+O(\sqrt{\# L}) .
\end{gathered}
$$

We now rewrite this penultimate sum as

$$
\begin{gathered}
\sum_{t \in \mathbb{G}_{m}(L)}\left(\sum_{x \in L, \lambda f(x)=t} \operatorname{Trace}\left(\operatorname{Frob}_{L, x} \mid \mathcal{F}\right)\right)\left(\sum_{y \in L, f(y)=t} \operatorname{Trace}\left(\operatorname{Frob}_{L, y} \mid \overline{\mathcal{F}}\right)\right)= \\
\sum_{(x, y) \in \mathbb{A}^{2}(L), \lambda f(x)=f(y) \neq 0} \operatorname{Trace}\left(F \operatorname{rob}_{L, x} \mid \mathcal{F}\right) \operatorname{Trace}\left(\operatorname{Frob}_{L, y} \mid \overline{\mathcal{F}}\right) .
\end{gathered}
$$

For $j: \mathbb{A}^{1}[1 / f] \subset \mathbb{A}^{1}$,if we add the $n^{2}$ terms

$$
\operatorname{Trace}\left(F r o b_{L, x} \mid j_{\star} \mathcal{F}\right) \operatorname{Trace}\left(F r o b_{L, y} \mid j_{\star} \overline{\mathcal{F}}\right)
$$

for the points $(x, y) \in \mathbb{A}^{2}(L)$ with $f(x)=f(y)=0$, i.e., for the $n^{2}$ points $\left(a_{i}, a_{j}\right)$, we only change our sum by $O(1)$ (and we don't change it at all if all the $\chi_{i}$ are nontrivial). So we end up with the estimate

$$
\begin{gathered}
\sum_{(x, y) \in \mathbb{A}^{2}(L), \lambda f(x)=f(y)} \operatorname{Trace}\left(\operatorname{Frob}_{L, x} \mid j_{\star} \mathcal{F}\right) \operatorname{Trace}\left(\operatorname{Frob}_{L, y} \mid j_{\star} \overline{\mathcal{F}}\right)= \\
=\alpha^{\operatorname{deg}(L / E)} \# L+O(\sqrt{\# L}) .
\end{gathered}
$$

We now explain how this estimate leads to a contradiction. Consider the affine curve of equation $\lambda f(x)=f(y)$ in $\mathbb{A}^{2}$. It is singular at the finitely many points $(a, b)$ which are pairs of critical points of $f$, i.e., $f^{\prime}(a)=f^{\prime}(b)=0$, such that $\lambda f(a)=f(b)$. It is nonsingular at each pair of zeroes $\left(a_{i}, a_{j}\right)$ of $f$. Replacing $E$ by a finite extension if necessary, we may further assume that each irreducible component of the curve $\lambda f(x)=f(y)$ over $E$ is geometrically irreducible (i.e., that each irreducible factor of $\lambda f(x)-f(y)$ in $E[x, y]$ remains irreducible in $\bar{k}[x, y])$. The penultimate sum is, up to an $O(1)$ term, the sum over the irreducible components $C_{j}$ of the curve $\lambda f(x)=f(y)$, of the sums

$$
\sum_{(x, y) \in \mathbb{C}_{j}(L)} \operatorname{Trace}\left(F_{\operatorname{rob}_{L, x}} \mid j_{\star} \mathcal{F}\right) \operatorname{Trace}\left(F_{r o b}{ }_{L, y} \mid j_{\star} \overline{\mathcal{F}}\right) \text {. }
$$

By the estimate for the sum, over the various $C_{j}$, of these sums, there is at least one irreducible component, call it $C$ for which this sum is not $O(\sqrt{\# L})$. The equation of any $C_{j}$ divides the polynomial $\lambda f(x)-f(y)$, whose highest degree term is $\lambda x^{n}-y^{n}$. Therefore the highest degree 
term of any divisor is a product of linear terms $\mu y-x$, with the various possible $\mu$ 's the $n$ 'th roots of $\lambda$. So an irreducible component $C_{i}$, given by a degree $d_{i}$ divisor of $\lambda f(x)-f(y)$, is finite flat of degree $d_{i}$ over the $y$-line (and over the $x$ line as well).

On the original curve $\lambda f(x)=f(y)$, for each $a_{j}$ there are $n$ points $\left(a_{j}, y\right)$ on the curve, namely $y=a_{i}$ for $i=1, \ldots, n$. On an irreducible component $C_{j}$, given by a degree $d_{j}$ divisor of $\lambda f(x)-f(y)$, there are at most $d_{j}$ values of $y$ such that $\left(a_{1}, y\right)$ lies on $C_{j}$. Each of these points is a smooth point of the original curve, so it lies only on the irreducible component $C_{j}$. As there are $n=\sum d_{j}$ points $\left(a_{1}, y\right)$ on the original curve, we must have exactly $d_{j}$ points on $C_{j}$ of the form $\left(a_{1}, y\right)$.

Now consider an irreducible component $C$ on which our sum is not $O(\sqrt{\# L})$. Let us denote by $\mathcal{C}$ the dense open set of the smooth locus of $C$ which, via $f$, lies over $\mathbb{G}_{m}$. The sum

$$
\sum_{(x, y) \in \mathcal{C}(L)} \operatorname{Trace}\left(\operatorname{Frob}_{L, x} \mid \mathcal{F}\right) \operatorname{Trace}\left(\operatorname{Frob}_{L, y} \mid \overline{\mathcal{F}}\right)
$$

differs only by $O(1)$ from the sum over $C$, so it too is not $O(\sqrt{\# L})$. In terms of the (restriction to $\mathcal{C}$ of the) lisse, pure of weight zero, lisse of rank one sheaf

$$
\mathcal{G}:=\otimes_{i=1}^{n} \mathcal{L}_{\chi_{i}\left(a_{i}-x\right)} \otimes_{i=1}^{n} \mathcal{L}_{\overline{\chi_{i}}\left(a_{i}-y\right)}
$$

on $\mathbb{A}^{2}[1 /(f(x) f(y))]$, this last sum is

$$
\sum_{(x, y) \in \mathcal{C}(L)} \operatorname{Trace}\left(\operatorname{Frob}_{L,(x, y)} \mid \mathcal{G}\right) .
$$

By the Lefschetz trace formula, this sum is

$$
\operatorname{Trace}\left(F_{r_{0}} \mid H_{c}^{2}(\mathcal{C} / \bar{k}, \mathcal{G})\right)-\operatorname{Trace}\left(F r o b_{L} \mid H_{c}^{1}(\mathcal{C} / \bar{k}, \mathcal{G})\right) .
$$

Because $\mathcal{G}$ is pure of weight zero and lisse of rank one, the $H_{c}^{2}$ is either zero or is one-dimensional and pure of weight two, and this second case only occurs when $\mathcal{G}$ is geometrically constant on $\mathcal{C}$. The $H_{c}^{1}$ is mixed of weight $\leq 1$. So the failure of an $O(\sqrt{\# L})$ estimate means that the $H_{c}^{2}$ is nonzero, and hence that $\mathcal{G}$ is geometrically constant on $\mathcal{C}$.

Suppose first that the equation of $C$ is of degre $d \geq 2$. Then there are $d$ points $\left(a_{1}, a_{i}\right)$ on $C$, at least one of which is of the form $\left(a_{1}, a_{i}\right)$ with $a_{i} \neq a_{1}$. The curve $C$ is finite etale over both the $x$-line and the $y$-line at the point $\left(a_{1}, a_{i}\right)$. So the functions $x-a_{1}$ and $y-a_{i}$ are each uniformizing parameters at this point. From the expression for $\mathcal{G}$, at the point $\left(a_{1}, a_{i}\right)$ on $C$ its inertia group representation is that of $\mathcal{L}_{\chi_{1}\left(x-a_{1}\right)} \otimes \mathcal{L}_{\overline{\chi_{i}}\left(y-a_{i}\right)}$. In other words, its inertia group representation at 
$\left(a_{1}, a_{i}\right)$ is the character $\chi_{1} / \chi_{i}$. But this character is nontrivial (because $\chi_{1}$ is a singleton), contradicting the geometric constance of $\mathcal{G}$ on $\mathcal{C}$.

It remains to treat the case in which the equation for $C$ is of degree one. In this case, the above argument still works unless the unique point on $C$ of the form $\left(a_{1}, y\right)$ has $y=a_{1}$. In this case, we use the fact that we have a second singleton, $\chi_{2}$. Using this singleton, we could still use the above argument unless the unique point on $C$ of the form $\left(a_{2}, y\right)$ has $y=a_{2}$. So we only need treat the case when both the points $\left(a_{1}, a_{1}\right)$ and $\left(a_{2}, a_{2}\right)$ lie on $C$. But in this case, the equation for $C$, being of degree one, must be $y=x$. But if $y-x$ divides $\lambda f(x)-f(y)$, we reduce $\bmod y-x$ to find that $(\lambda-1) f(x)=0$, and hence $\lambda=1$, contradiction.

\section{A preliminary estimate}

In this section, we continue with a squarefree monic $k$-polynomial $f$ of degree $n \geq 2, B:=k[X] /(f)$, and a character $\chi$ of $B^{\times}$. Over a finite extension $E / k$ where $f$ factors completely, say $f(X)=\prod_{i}\left(X-a_{i}\right)$, the lisse rank one sheaf $\mathcal{L}_{\chi(u-t)}$ on $\mathbb{A}^{1}[1 / f] / k$ becomes isomorphic to the sheaf $\otimes_{i=1}^{n} \mathcal{L}_{\chi_{i}\left(a_{i}-t\right)}$ on $\mathbb{A}^{1}[1 / f] / E$.

Theorem 4.1. Let $\chi$ be a character of $B^{\times}$whose constituent characters $\chi_{i}$ satisfy the following three conditions.

(1) The $\chi_{i}$ are pairwise distinct.

(2) The product $\prod_{i} \chi_{i}$ is nontrivial, (i.e., $\chi$ is nontrivial on $k^{\times}$).

(3) For at least one index $i, \chi_{i}^{n} \neq \prod_{i} \chi_{i}$.

Fix $\lambda \in k^{\times}$, and form the perverse sheaf

$$
N(\lambda, \chi):=[\lambda f]_{\star}\left(\mathcal{L}_{\chi(u-t)}\right)(1 / 2)[1]
$$

on $\mathbb{G}_{m} / k$. Then we have the following results.

(1) $N(\lambda, \chi)$ is geometrically irreducible, pure of weight zero, and lies in the Tannakian category $\mathcal{P}_{\text {arith }}$ in the sense of [Ka-CE]. It has generic rank $n$, Tannakian "dimension" $n-1$, and it has at most $2 n$ bad characters.

(2) $N(\lambda, \chi)$ is geometrically Lie-irreducible in $\mathcal{P}$.

(3) $N(\lambda, \chi)$ has $G_{\text {geom }}=G_{\text {arith }}=G L(n-1)$.

Proof. By Theorem 3.1 and the disjointness of the $\chi_{i}, N(\lambda, \chi)$ is geometrically irreducible. It visibly has generic rank $n$. As $n \geq 2$, it is not a Kummer sheaf, so, being geometrically irreducible, it lies in $\mathcal{P}$. Its Tannakian dimension is

$$
\chi_{c}\left(\mathbb{G}_{m} / \bar{k}, N(\lambda, \chi)\right)=-\chi_{c}\left(\mathbb{G}_{m} / \bar{k},[\lambda f]_{\star}\left(\mathcal{L}_{\chi(u-t)}\right)\right)=
$$




$$
=-\chi_{c}\left(\mathbb{A}^{1}[1 / f] / \bar{k}, \mathcal{L}_{\chi(u-t)}\right)=-\chi_{c}\left(\mathbb{A}^{1}[1 / f] / \bar{k}, \otimes_{i=1}^{n} \mathcal{L}_{\chi_{i}\left(a_{i}-t\right)}\right)=n-1 .
$$

Because $N(\lambda, \chi)$ has generic rank $n$, it has at most $2 n$ bad characters, namely those whose inverses occur in either its $I(0)$-representation or in its $I(\infty)$-representation.

On some dense open set $j: U \subset \mathbb{G}_{m},[\lambda f]_{\star}\left(\mathcal{L}_{\chi(u-t)}\right)$ is a lisse sheaf of rank $n$, which is pure of weight zero, hence $j^{\star} N(\lambda, \chi)$ is pure of weight zero (the Tate twist (1/2) offsets the shift [1]). By irreducibility $N(\lambda, \chi)$ must be the middle extension of $j^{\star} N(\lambda, \chi)$, cf.[BBD, 5.3.8], so remains pure of weight zero [BBD, 5.3.2]. Again by the disjointness of the $\chi_{i}$, part (3) of Theorem 3.1, together with [Ka-CE, Cor. 8.3], we get that $N(\lambda, \chi)$ is geometrically Lie-irreducible in $\mathcal{P}$.

It remains to explain why $N(\lambda, \chi)$ has $G_{\text {geom }}=G_{\text {arith }}=G L(n-1)$. Since we have a priori inclusions $G_{\text {geom }} \subset G_{\text {arith }} \subset G L(n-1)$, it suffices to prove that $G_{\text {geom }}=G L(n-1)$. The idea is to apply [Ka-CE, Thm. 17.1]. We may compute $G_{\text {geom }}$ after extension of scalars to $E$. Suppose that $\chi_{1}^{n} \neq \prod_{i} \chi_{i}$. The construction $M \mapsto M \otimes \mathcal{L}_{\overline{\chi_{1}}}$ induces a Tannakian isomorphism of $\langle N(\lambda, \chi)\rangle_{\text {arith }}$ with $\left\langle N(\lambda, \chi) \otimes \mathcal{L}_{\overline{\chi_{1}}}>_{\text {arith }}\right.$. So it suffices to prove that $N(\lambda, \chi) \otimes \mathcal{L}_{\overline{\chi_{1}}}$ has $G_{\text {geom }}=G L(n-1)$. By the disjointness assumption on the $\chi_{i}$, the trivial character $\mathbb{1}$ occurs exactly once in the $I(0)$-representation of $N(\lambda, \chi) \otimes \mathcal{L}_{\overline{\chi_{1}}}$. So by [Ka-CE, Thm. 17.1], it suffices to show that the trivial character does not occur in its $I(\infty)$ representation, or equivalently that $\chi_{1}$ does not occur in the $I(\infty)$ representation of $N(\lambda, \chi)$. This $I(\infty)$-representation is $[\lambda f]_{\star} \mathcal{L}_{\prod_{i} \chi_{i}}$, and $\mathcal{L}_{\chi_{1}}$ occurs in it if and only if $[\lambda f]^{\star}\left(\mathcal{L}_{\chi_{1}}\right)$ occurs in $\mathcal{L}_{\prod_{i} \chi_{i}}$. Because $\lambda f$ has degree $n$, the pullback $[\lambda f]^{\star}\left(\mathcal{L}_{\chi_{1}}\right)$ is geometrically isomorphic to $\mathcal{L}_{\chi_{1}^{n}}$ as $I(\infty)$-representation. So if $\chi_{1}^{n} \neq \prod_{i} \chi_{i}$, then $\mathcal{L}_{\chi_{1}}$ does not occur in the $I(\infty)$-representation $[\lambda f]_{\star} \mathcal{L}_{\prod_{i} \chi_{i}}$, and we conclude by applying [Ka-CE, Thm. 17.1] to $N(\lambda, \chi) \otimes \mathcal{L}_{\overline{\chi_{1}}}$.

Corollary 4.2. Let $\chi$ be a character of $B^{\times}$whose constituent characters $\chi_{i}$ satisfy the three conditions of the previous theorem. Suppose that $q:=\# k$ satisfies the inequality $\sqrt{q} \geq 1+2 n$. For each character $\rho$ of $k^{\times}$ which is good for $N(\lambda, \chi)$ (i.e., such that for $j: \mathbb{G}_{m} \subset \mathbb{P}^{1}$ the inclusion, the "forget supports" map gives an isomorphism $j_{!}\left(N(\lambda, \chi) \otimes \mathcal{L}_{\rho}\right) \cong$ $R j_{\star}\left(N(\lambda, \chi) \otimes \mathcal{L}_{\rho}\right)$, or equivalently, $\bar{\rho}$ does not occur in the local monodromy at either 0 or $\infty$ of $N(\lambda f, \chi))$, denote by $\theta_{k, \lambda f, \chi, \rho}$ the conjugacy class in $U(n-1)$ whose reversed characteristic polynomial is given by

$$
\operatorname{det}\left(1-T \theta_{k, \lambda f, \chi, \rho}\right)=\operatorname{det}\left(1-\operatorname{TFrob}_{k} \mid H_{c}^{0}\left(\mathbb{G}_{m} / \bar{k}, N(\lambda, \chi) \otimes \mathcal{L}_{\rho}\right) .\right.
$$


Let $\Lambda$ be a nontrivial irreducible representation of $U(n-1)$ which occurs in $s t d^{\otimes a} \otimes\left(s t d^{\vee}\right)^{\otimes b}$. Then we have the estimate

$$
\begin{gathered}
\left|\sum_{\rho \in \operatorname{Good}(k, \lambda f, \chi)} \operatorname{Trace}\left(\Lambda\left(\theta_{k, \lambda f, \chi, \rho}\right)\right)\right| \\
\leq(\# \operatorname{Good}(k, \lambda f, \chi)) 2(a+b+1)(2 n)^{a+b} / \sqrt{q} .
\end{gathered}
$$

Proof. By Theorem 4.1, $N(\lambda, \chi)$ has $G_{\text {geom }}=G_{\text {arith }}=G L(n-1)$. So this is [Ka-CE, Remark 7.5 and the proof of Theorem 28.1], applied to $N:=N(\lambda, \chi)$ with the constant $C$ there, an upper bound for each of the generic rank, the number of bad characters, and the Tannakian dimension of $N$, taken to be $2 n$.

The interest of this Corollary is that the (trivial) Leray spectral sequence for $[\lambda f]$ ! gives a Frob $_{k}$-isomorphism of cohomology groups

$$
\begin{gathered}
H_{c}^{0}\left(\mathbb{G}_{m} / \bar{k}, N(\lambda, \chi) \otimes \mathcal{L}_{\rho}\right) \cong H_{c}^{0}\left(\mathbb{A}^{1}[1 / f] / \bar{k}, \mathcal{L}_{\chi(u-t)} \otimes \mathcal{L}_{\rho(\lambda f(t))}(1 / 2)[1]\right)= \\
=H_{c}^{1}\left(\mathbb{A}^{1}[1 / f] / \bar{k}, \mathcal{L}_{\chi(u-t)} \otimes \mathcal{L}_{\rho(\lambda f(t))}\right)(1 / 2) .
\end{gathered}
$$

By Lemma 2.1, $\operatorname{Norm}_{B / k}(u-t)=(-1)^{n} f(t)$. So if we denote by $\rho_{\text {Norm }}$ the character of $B^{\times}$given by

$$
\rho_{\text {Norm }}:=\rho \circ \operatorname{Norm}_{B / k},
$$

then $\mathcal{L}_{\rho\left((-1)^{n} f(t)\right)}$ is $\mathcal{L}_{\rho_{\text {Norm }}(u-t)}$, and the conjugacy class $\theta_{k,(-1)^{n} f, \chi, \rho}$ is none other than the conjugacy class $\theta_{k, f, \chi \rho_{\text {Norm }}}$ of the Introduction.

\section{THE EQUidistribution THEOREM}

We continue with a squarefree monic $k$-polynomial $f$ of degree $n \geq 2$, $B:=k[X] /(f)$, and a character $\chi$ of $B^{\times}$. Over a finite extension $E / k$ where $f$ factors completely, say $f(X)=\prod_{i}\left(X-a_{i}\right)$, the lisse rank one sheaf $\mathcal{L}_{\chi(u-t)}$ on $\mathbb{A}^{1}[1 / f] / k$ becomes isomorphic to the sheaf $\otimes_{i=1}^{n} \mathcal{L}_{\chi_{i}\left(a_{i}-t\right)}$ on $\mathbb{A}^{1}[1 / f] / E$.

Let us say that $\chi$ is "totally ramified" (what we called "as ramified as possible" in the Introduction) if each $\chi_{i}$ and the product $\prod_{i} \chi_{i}$ are all nontrivial. In view of Lemma $2.2, \chi$ is totally ramified if and only if the group $H_{c}^{1}\left(\mathbb{A}^{1}[1 / f] / \bar{k}, \mathcal{L}_{\chi(u-t)}\right)$ is pure of weight one, or equivalently if and only if the group $H_{c}^{0}\left(\mathbb{A}^{1}[1 / f] / \bar{k}, \mathcal{L}_{\chi(u-t)}(1 / 2)[1]\right)$ is pure of weight zero, in which case it has dimension $n-1$.

Let us say that a totally ramified $\chi$ is "generic" if, in addition to being totally ramified, its constituent characters $\chi_{i}$ satisfy the three conditions of Theorem 4.1. We denote by

$$
\operatorname{TotRam}(k, f), \text { resp. TotRamGen }(k, f)
$$


the sets of totally ramified (respectively totally ramified and generic) characters of $B^{\times}$.

Lemma 5.1. Let $\chi$ be a totally ramified character of $B^{\times}$. Let $\rho$ be a character of $k^{\times}$which is good for $N\left((-1)^{n} f, \chi\right)$. Then the product character $\chi \rho_{\text {Norm }}$ is totally ramified. Moreover, $\chi$ is generic if and only if $\chi \rho_{\text {Norm }}$ is generic.

Proof. Indeed, if geometrically we have $\mathcal{L}_{\chi(u-t)} \cong \otimes_{i=1}^{n} \mathcal{L}_{\chi_{i}\left(a_{i}-t\right)}$, then $\mathcal{L}_{\chi \rho_{\text {Norm }}(u-t)} \cong \otimes_{i=1}^{n} \mathcal{L}_{\chi_{i} \rho\left(a_{i}-t\right)}$; we view $\rho$ and the $\chi_{i}$ as characters of $\pi_{1}^{\text {tame }}\left(\mathbb{G}_{m} / \bar{k}\right)$, to make sense of the products $\chi_{i} \rho$. Alternatively, if $f$ splits over $E$, think of $\rho$ as the character $x \mapsto \rho\left(\operatorname{Norm}_{E / k}(x)\right)$ of $E^{\times}$. Thus the constituent characters of $\chi \rho_{\text {Norm }}$ are the $\chi_{i} \rho$. That $\rho$ is good for $N\left((-1)^{n} f, \chi\right)$ means precisely $\bar{\rho}$ does not occur in the local monodromy of $N\left((-1)^{n} f, \chi\right)$ at either 0 or $\infty$. Its absence at 0 is the nontriviality of each $\chi_{i} \rho$. Its absence at $\infty$ is that $\rho^{n} \prod_{i} \chi_{i}$ is nontrivial, i.e., that $\prod_{i}\left(\chi_{i} \rho\right)$ is nontrivial. Thus $\chi \rho_{\text {Norm }}$ is totally ramified. If in addition $\chi$ is generic, say $\chi_{1}^{n} \neq \prod_{i} \chi_{i}$, then $\left(\chi_{1} \rho\right)^{n} \neq \prod_{i}\left(\chi_{i} \rho\right)$, and hence $\chi \rho_{\text {Norm }}$ is generic as well. Conversely, if $\chi$ is totally ramified and $\chi \rho_{\text {Norm }}$ is totally ramified and generic, then $\bar{\rho}$ is good for $N\left((-1)^{n} f, \chi \rho_{\text {Norm }}\right)$, and so by the previous argument $\chi$ is totally ramified and generic.

We now combine this lemma with Corollary 4.2 to get a result concerning those conjugacy classes $\theta_{k, f, \chi}$ of the Introduction whose $\chi$ is totally ramified and generic.

Corollary 5.2. Suppose $\sqrt{q} \geq 1+2 n$. Let $\Lambda$ be a nontrivial irreducible representation of $U(n-1)$ which occurs in std $d^{\otimes a} \otimes\left(s t d^{\vee}\right)^{\otimes b}$. Then we have the estimate

$$
\begin{gathered}
\left|\sum_{\chi \in \operatorname{TotRamGen}(k, f)} \operatorname{Trace}\left(\Lambda\left(\theta_{k, f, \chi}\right)\right)\right| \\
\leq\left(\# \operatorname{TotRamGen}(k, f) 2(a+b+1)(2 n)^{a+b} / \sqrt{q} .\right.
\end{gathered}
$$

Proof. Let us say that two totally ramified generic characters $\chi$ and $\chi^{\prime}$ of $B^{\times}$are equivalent if $\chi^{\prime}=\chi \rho_{\text {Norm }}$ for some (necessarily unique) character $\rho$ of $k^{\times}$. Break the terms of the sum into equivalence classes. The sum over the equivalence class of $\chi$ is precisely the sum bounded by Corollary 4.2 , with $\lambda$ there taken to be $(-1)^{n}$.

Our final task is to infer from this estimate an estimate for the sum over all $\chi$ in $\operatorname{Tot} \operatorname{Ram}(k, f)$. For this, we now turn to giving upper and lower bounds for \#TotRam $(k, f)$ and for \#TotRamGen $(k, f)$. We define three monic integer polynomials of degree $n$,

$$
P_{\text {all }, n}(X):=X^{n}-1,
$$




$$
P_{T R, n}(X):=(X-2)^{n}-\sum_{0 \leq i \leq n-1} X^{i}
$$

and

$$
P_{T R G, n}(X):=(X-1-n)^{n}+(X-2)^{n}-X^{n}+1-n \sum_{0 \leq i \leq n-1} X^{i} .
$$

Lemma 5.3. For $q:=\# k$, we have the (trivial) estimate

$$
\# \operatorname{TotRam}(k, f) \leq P_{\mathrm{all}, n}(q)=q^{n}-1 .
$$

Proof. Indeed, $B^{\times}$is a subset of $B \backslash\{0\}$, whose cardinality is $q^{n}-1$, so $q^{n}-1$ is an upper bound for the total number of characters of $B^{\times}$.

Lemma 5.4.

$$
\# \operatorname{Tot} \operatorname{Ram}(k, f) \geq P_{T R, n}(q)=(q-2)^{n}-\sum_{0 \leq i \leq n-1} q^{i},
$$

and

$$
\#\left(\left\{\operatorname{char}^{\prime} \text { s of } B^{\times}\right\} \backslash \operatorname{TotRam}(k, f)\right) \leq q^{n}-1-(q-2)^{n}+\sum_{0 \leq i \leq n-1} q^{i} .
$$

Proof. Factor $f$ as the product of $k$-irreducible monic polynomials $P_{i}$ of degree $d_{i}$. Thus $n=\sum_{i} d_{i}$, and $\# B^{\times}=\prod_{i}\left(q^{d_{i}}-1\right) \geq(q-1)^{n}$. So there are at least $(q-1)^{n}$ characters $\chi$ of $B^{\times}$. We now count the characters which violate or satisfy the two conditions of being totally ramified.

Since $k^{\times} \subset B^{\times}$, the restriction map on characters is surjective. So the condition that $\chi \mid k^{\times}$be nontrivial disqualifies $\# B^{\times} /(q-1)=$ $\left(\prod_{i}\left(q^{d_{i}}-1\right)\right) /(q-1)$ of them.

The condition that each constituent character $\chi_{i}$ is nontrivial is equivalent to the condition that when we write $\chi$ as the product of characters $\chi_{P_{i}}$ of the factors $\left(k[X] /\left(P_{i}\right)\right)^{\times}$, each $\chi_{P_{i}}$ is nontrivial. So there are $\prod_{i}\left(q^{d_{i}}-2\right)$ choices of $\chi$ which satisfy this condition. If we now omit the ones which are trivial on $k^{\times}$, we are left with at least

$$
\prod_{i}\left(q^{d_{i}}-2\right)-\left(\prod_{i}\left(q^{d_{i}}-1\right)\right) /(q-1)
$$

characters which are totally ramified. From the inequalities $q^{d}-2 \geq$ $(q-2)^{d}$ and $\prod_{i}\left(q^{d_{i}}-1\right) \leq q^{n}-1$ we get

$$
\begin{aligned}
\# \operatorname{Tot} \operatorname{Ram}(k, f) & \geq \prod_{i}\left(q^{d_{i}}-2\right)-\left(\prod_{i}\left(q^{d_{i}}-1\right)\right) /(q-1) \geq \\
& \geq(q-2)^{n}-\sum_{0 \leq i \leq n-1} q^{i} .
\end{aligned}
$$


Combining this with the previous lemma, we get the asserted upper bound for the number of characters of $B^{\times}$which are not totally ramified.

Lemma 5.5. For $q \geq n+1$, we have the estimate

$$
\begin{gathered}
\text { \#TotRamGen }(k, f) \geq P_{T R G, n}(q)= \\
=(q-1-n)^{n}+(q-2)^{n}-q^{n}+1-n \sum_{0 \leq i \leq n-1} q^{i} .
\end{gathered}
$$

Proof. We now count the characters which violate or satisfy the two additional condtions which make a totally ramified character generic

We first turn to the condition that for at least one of the $\chi_{i}, \chi_{i}^{n} \neq$ $\prod_{i} \chi_{i}$. Suppose first that $f$ is itself irreducible. Then $\chi$ is a character of the field $B^{\times} \cong \mathbb{F}_{q^{n}}^{\times}$, and its constituent characters $\chi_{1}, \ldots, \chi_{n}$ are the characters $\chi, \chi^{q}, \ldots, \chi^{q^{n-1}}$. The condition that $\chi^{n} \neq \prod_{i} \chi_{i}$ is the condition that $\chi^{n} \neq \chi^{1+q+\ldots+q^{n-1}}$, which disqualifies at most $1+q+$ $\ldots+q^{n-1}-n$ possible $\chi$.

If $f$ is not irreducible, let $P$ be an irreducible factor of some degree $d<n$, and $\chi_{P}$ the $P$-constituent of $\chi$. The constituents of $\chi_{P}$ as character of $(k[X] /(P))^{\times}$are $\chi_{P}, \chi_{P}^{q}, \ldots, \chi_{P}^{q^{d-1}}$. Think of these as the first $d$ constituents of $\chi$. We can be sure that there is some choice of index $j \in[1, d]$ such that $\chi_{j}^{n} \neq \prod_{i} \chi_{i}$ if we have

$$
\prod_{1 \leq j \leq d} \chi_{j}^{n} \neq\left(\prod_{i} \chi_{i}\right)^{d}
$$

This is the condition that

$$
\chi_{P}^{(n-d)\left(1+q+\ldots+q^{d-1}\right)} \neq\left(\prod_{d+1 \leq i \leq n} \chi_{i}\right)^{d} .
$$

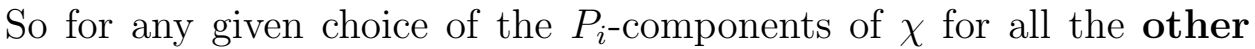
irreducible factors $P_{i}$ of $f$, at most $(n-d)\left(1+q+\ldots+q^{d-1}\right)$ characters $\chi_{P}$ are disqualified. So the total number of characters $\chi$ which fail this second condition is at most $(n-d)\left(1+q+\ldots+q^{d-1}\right) \prod_{P_{i} \neq P}\left(q^{d_{i}}-1\right)$. From the inequality

$$
\begin{gathered}
(n-d)\left(1+q+\ldots+q^{d-1}\right) \prod_{P_{i} \neq P}\left(q^{d_{i}}-1\right)=(n-d)\left(\prod_{\text {all } \mathrm{P}_{\mathrm{i}}}\left(q^{d_{i}}-1\right)\right)(q-1) \\
\leq(n-1)\left(q^{n}-1\right) /(q-1)
\end{gathered}
$$

we see that the in either case, $f$ irreducible or not, there are at most

$$
(n-1)\left(\sum_{0 \leq i \leq n-1} q^{i}\right)
$$


characters $\chi$ of $B^{\times}$which violate this first condition.

We now turn to the condition that the constituents $\chi_{i}$ be all distinct. Again we factor $f$, and this time collect the factors according to their degrees. Suppose that there are $e_{i}$ factors $P_{d_{i}, j}, j=1, \ldots, e_{i}$ whose degrees are $d_{i}$. The first condition for distinctness is that for each $P_{d_{i}, j}$-component $\chi_{P_{d_{i}, j}}$ the $d_{i}$ characters $\chi_{P_{d_{i}, j}}^{q^{i}}$ for $0 \leq i \leq d_{i}-1$ are all distinct, or in other words that the orbit of $\chi_{P_{d_{i}, j}}$ under the $q$ 'th power map has full length $d_{i}$, rather than some proper divisor of $d_{i}$. The characters of $\mathbb{F}_{q^{d_{i}}}^{\times}$whose orbit length is a proper divisor of $d_{i}$ are those which come from (by composition with the relative norm) characters of subfields $\mathbb{F}_{q^{r}}$ for some proper divisor $r$ of $d_{i}$. So the number of such short-orbit characters is at most $\sum_{r \mid d_{i}, r<d_{i}}\left(q^{r}-1\right)$, and this is trivially bounded by

$\sum_{r \mid d_{i}, r<d_{i}}\left(q^{r}-1\right) \leq-1+\sum_{r \mid d_{i}, r<d_{i}} q^{r} \leq-1+\sum_{1 \leq r \leq d_{i} / 2} q^{r} \leq-1+\left[d_{i} / 2\right] q^{\left[d_{i} / 2\right]}$.

So the number of full-orbit characters of $\mathbb{F}_{q^{d_{i}}}^{\times}$is at least

$$
q^{d_{i}}-\left[d_{i} / 2\right] q^{\left[d_{i} / 2\right]} \geq q^{d_{i}}-q^{d_{i}-1} .
$$

Suppose now that for each irreducible factor $P_{d_{i}, j}$ of $f$, we have chosen a full-orbit (i.e., orbit length $d_{i}$ ) character. For irreducibles of different degrees, there can be no equality of their constituent characters, because the orbit-lengths are different. If there are $e_{i} \geq 2$ irreducible factors of the same degree $d_{i}$, say $P_{d_{i}, 1}, \ldots, P_{d_{i}, e_{i}}$, then we may choose $\chi_{P_{d_{i}, 1}}$ to be any of the at least $q^{d_{i}}-q^{d_{i}-1}$ full-orbit characters of $\mathbb{F}_{q^{d_{i}}}^{\times}$. Then we must choose $\chi_{P_{d_{i}, 2}}$ to be a full-orbit character of $\mathbb{F}_{q^{d_{i}}}^{\times}$which does lie in the orbit of $\chi_{P_{d_{i}, 1}}$, thus excluding $d_{i}$ possible full-orbit characters. Continuing in this way, we see that there are at least

$$
\prod_{d_{i} \text { which occur }}\left(\prod_{j=0}^{e_{i}-1}\left(q^{d_{i}}-q^{d_{i}-1}-j d_{i}\right)\right)
$$

characters $\chi$ of $B^{\times}$all of whose constituents are distinct.

Because $q \geq n+1$, each factor $\left(q^{d_{i}}-q^{d_{i}-1}-j d_{i}\right)$ satisfies

$$
\left(q^{d_{i}}-q^{d_{i}-1}-j d_{i}\right) \geq\left(q^{d_{i}}-q^{d_{i}-1}-n\right) \geq(q-1-n)^{d_{i}} .
$$

[For the last inequality, write $q=X+n+1$; then we are saying that $(X+n+1)^{d-1}(X+n) \geq X^{d}+n$, which obviously holds for $X \geq 0$ and $d \geq 1$.] Thus for $q \geq n+1$, there are at least

$$
(q-1-n)^{n}
$$

characters $\chi$ of $B^{\times}$all of whose constituents are distinct. 
Removing from these those which violate the first condition, we are left with at least

$$
(q-1-n)^{n}-(n-1)\left(\sum_{0 \leq i \leq n-1} q^{i}\right)
$$

characters which, if totally ramified, are also generic. We have already seen that at most

$$
q^{n}-1-(q-2)^{n}+\sum_{0 \leq i \leq n-1} q^{i}
$$

characters fail to be totally ramified. Taking (some of) these away, we end up with at least

$$
\begin{gathered}
(q-1-n)^{n}-(n-1)\left(\sum_{0 \leq i \leq n-1} q^{i}\right)-\left(q^{n}-1-(q-2)^{n}+\sum_{0 \leq i \leq n-1} q^{i}\right)= \\
=(q-1-n)^{n}+(q-2)^{n}-q^{n}+1-n \sum_{0 \leq i \leq n-1} q^{i}
\end{gathered}
$$

characters which are totally ramified and generic.

Lemma 5.6. We have the estimate

$$
\begin{gathered}
\text { \# }\left(\text { TotRam }(k, f) \backslash \text { TotRamGen }(k, f) \leq P_{\mathrm{all}, n}(q)-P_{T R G, n}(q)=\right. \\
=(q-1-n)^{n}+(q-2)^{n}-2 q^{n}+2-n \sum_{0 \leq i \leq n-1} q^{i} .
\end{gathered}
$$

Proof. Combine Lemmas 5.3 and 5.5.

Lemma 5.7. There exists a real constant $C_{n}$ such that for $q \geq C_{n}$, we have

$$
P_{\mathrm{all}, n}(q)-P_{T R G, n}(q) \leq P_{T R G, n}(q) / \sqrt{q}
$$

Proof. The difference $P_{\text {all, } n}(X)-P_{T R G, n}(X)$ is a real polynomial of degree $n-1$, while $P_{T R G, n}(X)$ is a real polynomial which is monic of degree $n$.

Theorem 5.8. Suppose $q \geq C_{n}$ and $\sqrt{q} \geq 1+2 n$. Let $\Lambda$ be a nontrivial irreducible representation of $U(n-1)$ which occurs in $s t d^{\otimes a} \otimes\left(s t d^{\vee}\right)^{\otimes b}$. Then we have the estimate

$$
\begin{gathered}
\left|\sum_{\chi \in \operatorname{TotRam}(k, f)} \operatorname{Trace}\left(\Lambda\left(\theta_{k, f, \chi}\right)\right)\right| \\
\leq(\# \operatorname{TotRamGen}(k, f)) 4(a+b+1)(2 n)^{a+b} / \sqrt{q} .
\end{gathered}
$$


Proof. We break the sum into two pieces, the sum over $\chi \in \operatorname{Tot} \operatorname{RamGen}(k, f)$, and the sum over $\chi \in \operatorname{Tot} \operatorname{Ram}(k, f) \backslash \operatorname{TotRamGen}(k, f)$. By Corollary 5.2 , the absolute value of the first sum is bounded by

$$
(\# \text { TotRamGen }(k, f)) 2(a+b+1)(2 n)^{a+b} / \sqrt{q} .
$$

The second sum has at most

$$
P_{\mathrm{all}, n}(q)-P_{T R G, n}(q) \leq P_{T R G, n}(q) / \sqrt{q} \leq(\# \operatorname{TotRamGen}(k, f)) / \sqrt{q}
$$

terms, each of which, being the trace of a unitary conjugacy class in a representation of dimension at most $(n-1)^{a+b}$, is bounded in absolute value by $(n-1)^{a+b}$. So the absolute value of the second sum is bounded by

$$
(\# \text { TotRamGen }(k, f))(n-1)^{a+b} / \sqrt{q},
$$

which is less than the upper bound for the first sum. So doubling the upper bound for the first sum is safe.

Corollary 5.9. Suppose $q \geq C_{n}$ and $\sqrt{q} \geq 1+2 n$. Let $\Lambda$ be a nontrivial irreducible representation of $U(n-1)$ which occurs in std ${ }^{\otimes a} \otimes\left(s t d^{\vee}\right)^{\otimes b}$. Then we have the estimate

$$
\begin{gathered}
\left|(1 / \# \operatorname{TotRam}(k, f)) \sum_{\chi \in \operatorname{TotRam}(k, f)} \operatorname{Trace}\left(\Lambda\left(\theta_{k, f, \chi}\right)\right)\right| \\
\leq 4(a+b+1)(2 n)^{a+b} / \sqrt{q} .
\end{gathered}
$$

Proof. Indeed, \#TotRamGen $(k, f) \leq \# \operatorname{TotRam}(k, f)$.

Thus we obtain our target result.

Theorem 5.10. Fix an integer $n \geq 2$ and a sequence of data $\left(k_{i}, f_{i}\right)$ with $k_{i}$ a finite field (of possibly varying characteristic) and $f_{i}(X) \in$ $k_{i}[X]$ squarefree of degree $n$. If $\# k_{i}$ is archimedeanly increasing to $\infty$, the collections of conjugacy classes

$$
\left\{\theta_{k_{i}, f_{i}, \chi}\right\}_{\chi \in \operatorname{TotRam}\left(k_{i}, f_{i}\right)}
$$

become equidistributed in $U(n-1)^{\#}$ as $\# k_{i} \rightarrow \infty$.

\section{Appendix: The case of "EVen" Characters}

We continue to work with a squarefree monic polynomial $f(X) \in$ $k[X]$ of degree $n \geq 2$, and the $k$-algebra $B:=k[X] /(f(X))$. We say that a character $\chi$ of $B^{\times}$is even if it is trivial on $k^{\times}$(viewed as a subgroup of $B^{\times}$). 
Lemma 6.1. The character $\chi$ is even if and only if $\mathcal{L}_{\chi(u-t)}$ is lisse at $\infty$ (more precisely, if and only if, denoting by $j: \mathbb{A}^{1}[1 / f] \subset \mathbb{P}^{1}$ the inclusion, the middle extension sheaf $j_{\star} \mathcal{L}_{\chi(u-t)}$ on $\mathbb{P}^{1}$ is lisse at $\left.\infty\right)$. Moreover, for even $\chi$ we have the formula

$$
\operatorname{Trace}\left(\operatorname{Frob}_{k, \infty} \mid j_{\star} \mathcal{L}_{\chi(u-t)}\right)=1 .
$$

Proof. The first assertion is immediate from the geometric isomorphism of $\mathcal{L}_{\chi(u-t)}$ with the tensor product $\otimes_{i=1}^{n} \mathcal{L}_{\chi_{i}\left(a_{i}-t\right)}$, together with Lemma 2.3. For the second assertion, we argue as follows. We have a morphism $\mathbb{G}_{m} \rightarrow \mathbb{B}^{\times}$given by $t \mapsto 1 / t$. The corresponding pullback sheaf $\mathcal{L}_{\chi(1 / t)}$ on $\mathbb{G}_{m}$ is trivial, i.e., isomorphic to the constant sheaf $\overline{\mathbb{Q}}$, precisely because $\chi$ is trivial on $k^{\times}$. So on $\mathbb{G}_{m}[1 / f]$, we have arithmetic isomorphisms

$$
\mathcal{L}_{\chi(u-t)} \cong \mathcal{L}_{\chi(u-t)} \otimes \mathcal{L}_{\chi(1 / t)} \cong \mathcal{L}_{\chi(u / t-1)} .
$$

In terms of the uniformizing parameter $s:=1 / t$ at $\infty$, we have $\mathcal{L}_{\chi(u-t)} \cong$ $\mathcal{L}_{\chi(s u-1)}$. Extending $\mathcal{L}_{\chi(s u-1)}$ across $\infty$, i.e., across $s=0$, by direct image, we get

$$
\operatorname{Trace}\left(\operatorname{Frob}_{k, \infty} \mid j_{\star} \mathcal{L}_{\chi(u-t)}\right)=\operatorname{Trace}\left(F r o b_{k, 0} \mid j_{\star} \mathcal{L}_{\chi(s u-1)}\right)=\chi(-1)=1,
$$

the last equality because, once again, $\chi$ is trivial on $k^{\times}$.

Let us say that an even character $\chi$ is totally ramified if, in the geometric isomorphism

$$
\mathcal{L}_{\chi(u-t)} \cong \otimes_{i=1}^{n} \mathcal{L}_{\chi_{i}\left(a_{i}-t\right)}
$$

each $\chi_{i}$ is nontrivial. The we have the following lemma, analogous to Lemma 2.5

Lemma 6.2. The even character $\chi$ is totally ramified if and only if the group $H_{c}^{1}\left(\mathbb{P}^{1}[1 / f] \otimes_{k} \bar{k}, j_{\star} \mathcal{L}_{\chi(u-t)}\right)$ is pure of weight one, in which case $H_{c}^{1}$ has dimension $n-2$, and $H_{c}^{2}=0$.

Let us denote by TotRamEven $(k, f)$ the set of even characters of $B^{\times}$ which are totally ramified. Attached to each $\chi \in \operatorname{TotRamEven}(k, f)$, we have a conjugacy class $\theta_{k, f, \chi} \in U(n-2)^{\#}$, defined by its reversed characteristic polynomial via the equation

$$
\operatorname{det}\left(1-T \sqrt{\# k} \theta_{k, f, \chi}\right)=\operatorname{det}\left(1-\operatorname{TFrob}_{k} \mid H_{c}^{1}\left(\mathbb{P}^{1}[1 / f] \otimes_{k} \bar{k}, j_{\star} \mathcal{L}_{\chi(u-t)}\right)\right) \text {. }
$$

Keating and Rudnick, in a personal communication, made the following conjecture, the "even" version of Theorem 5.10.

Conjecture 6.3. Fix an integer $n \geq 3$ and a sequence of data $\left(k_{i}, f_{i}\right)$ with $k_{i}$ a finite field (of possibly varying characteristic) and $f_{i}(X) \in$ 
$k_{i}[X]$ squarefree of degree $n$. If $\# k_{i}$ is archimedeanly increasing to $\infty$, the collections of conjugacy classes

$$
\left\{\theta_{k_{i}, f_{i}, \chi}\right\}_{\chi \in \operatorname{TotRamEven}\left(k_{i}, f_{i}\right)}
$$

become equidistributed in $U(n-2)^{\#}$ as $\# k_{i} \rightarrow \infty$.

At present, we can prove this only under the additional (and highly artificial) hypothesis that each $f_{i}(X) \in k_{i}[X]$ has a zero in $k_{i}$.

Theorem 6.4. Fix an integer $n \geq 3$ and a sequence of data $\left(k_{i}, f_{i}\right)$ with $k_{i}$ a finite field (of possibly varying characteristic) and $f_{i}(X) \in k_{i}[X]$ squarefree of degree $n$. Suppose each $f_{i}$ has a zero in $k_{i}$. If $\# k_{i}$ is archimedeanly increasing to $\infty$, the collections of conjugacy classes

$$
\left\{\theta_{k_{i}, f_{i}, \chi}\right\}_{\chi \in \operatorname{TotRamEven}\left(k_{i}, f_{i}\right)}
$$

become equidistributed in $U(n-2)^{\#}$ as $\# k_{i} \rightarrow \infty$.

Proof. Replacing each $f_{i}$ by an additive translate $X \mapsto X+a_{i}$ of itself, we reduce to the case when each $f_{i}$ is of the form $f_{i}(X)=X g_{i}(X)$, with $g_{i} \in k_{i}[X]$ squarefree and having $g_{i}(0) \neq 0$.

The idea is that the theorem is a consequence of a (slight variant of) Theorem 5.10, applied to the $g_{i}$. To explain this, let us fix a finite field $k$, a squarefree monic $g(X) \in k[X]$ of degree $n-1$ with $g(0) \neq 0$, and put $f(X):=X g(X)$. Let us write $B_{f}:=k[X] /(f(X)), B_{g}:=$ $k[X] /(g(X)), B_{X}:=k[X] /(X) \cong k$. Then

$$
B_{f} \cong k \times B_{g}
$$

For $P(X)$ a monic irreducible in $k[X]$ which is prime to $f$, the image of $P(X)$ in $B_{f}^{\times}$is, via this isomorphism, the pair

$$
(P(0), P \bmod g)=\left(\text { the scalar } P(0) \in k^{\times}\right) \times(1, P / P(0) \bmod g) .
$$

For an even character $\chi_{f}$ of $B_{f}^{\times}$, with components $\chi_{X}, \chi_{g}$, we therefore have

$$
\chi_{f}(P \bmod f)=\chi_{f}(1, P / P(0) \bmod g)=\chi_{g}(P / P(0)) .
$$

If $\chi_{f}$ lies in TotRamEven $(k, f)$ then $\chi_{X}$ is nontrivial, each constituent chararcter $\chi_{i}$ of $\chi_{g}$ is nontrivial, and, by the evenness of $\chi_{f}$, the restriction of $\chi_{g}$ to $k^{\times}$is the inverse of the nontrivial character $\chi_{X}$. In other words, $\chi_{g} \in \operatorname{Tot} \operatorname{Ram}(k, g)$. Conversely, given $\chi_{g} \in \operatorname{Tot} \operatorname{Ram}(k, g)$, define $\chi_{X}$ to be the restriction to $k^{\times}$of $1 \chi_{g}$; then the pair $\left(\chi_{X}, \chi_{g}\right)$ taken to be $\chi_{f}$ lies in TotRamEven $(k, f)$.

For $P(X)=X-t$ a linear irreducible, and $\chi_{f}$ even, we have

$$
\chi_{f}(X-t)=\chi_{g}((X-t) /(-t))=\chi_{g}(1-X / t) .
$$


Exactly as in section 2 of this paper, we find an arithmetic isomorphism on $\mathbb{A}^{1}[1 / f]=\mathbb{G}_{m}[1 / g]$,

$$
\mathcal{L}_{\chi_{f}(u-t)} \cong \mathcal{L}_{\chi_{g}(1-u / t)}
$$

In terms of the parameter $s:=1 / t$ on $\mathbb{G}_{m}$, and the palindrome $g^{\text {pal }}(s):=$ $s^{\operatorname{deg}(g)} g(t)$ of $g$, our sheaf becomes $\mathcal{L}_{\chi_{g}(1-u s)}$ on $\mathbb{G}_{m}\left[1 / g^{\text {pal }}\right]$, and has an obvious lisse extension across $s=0$ to the sheaf $\mathcal{L}_{\chi_{g}(1-u s)}$ on $\mathbb{A}^{1}\left[1 / g^{\text {pal }}\right]$. [N.B. Here the $u$ is still the image of $X$ in $B_{g}$, and $\chi_{g}$ is our character of $B_{g}^{\times}$. But it is the zeroes of $g^{\text {pal }}(s)$ we must avoid.]

We now define conjugacy classes $\Theta_{k, g, \chi_{g}} \in U(n-2)^{\#}$, for each $\chi_{g} \in$ $\operatorname{Tot} \operatorname{Ram}(k, g)$, through their reversed characteristic polynomials

$\operatorname{det}\left(1-T \sqrt{\# k} \Theta_{k, g, \chi_{g}}\right)=\operatorname{det}\left(1-\right.$ TFrob $_{k} \mid H_{c}^{1}\left(\mathbb{A}^{1}\left[1 / g^{p a l}\right] \otimes_{k} \bar{k}, \mathcal{L}_{\chi_{g}(1-u s)}\right)$.

With these preliminaries out of the way, we see that we have reduced Theorem 6.4 to the variant of Theorem 5.10 for the conjugacy classes $\left\{\Theta_{k, g, \chi_{g}}\right\}_{\chi_{g} \in \operatorname{TotRam}(k, g)}$. To prove this variant, we repeat the proof of Theorem 5.10, but looking at the direct image by $g^{\text {pal }}$ of $\mathcal{L}_{\chi_{g}(1-u s)}$ (rather than looking at the direct image by $(-1)^{\operatorname{deg}(g)} g$ of $\mathcal{L}_{\chi_{g}(u-t)}$, as we did in proving Theorem 5.10).

\section{REFERENCES}

[BBD] Beilinson, A., Bernstein, J., and Deligne, P., Faisceaux pervers. (entire contents of) Analyse et topologie sur les espaces singuliers, I (Conférence de Luminy, 1981), 5-171, Astérisque, 100, Soc. Math. France, Paris, 1982.

[De-Weil II] Deligne, P., La conjecture de Weil II. Publ. Math. IHES 52 (1981), 313-428.

[Ka-CE] Katz, N., Convolution and Equidistribution: Sato-Tate Theorems for Finite-Field Mellin Transforms. Annals of Mathematics Studies, 180. Princeton Univ. Press, Princeton, NJ, 2012.

[Ka-TLFM] Katz, N., Twisted L-functions and monodromy. Annals of Math. Studies, 150. Princeton Univ. Press, Princeton, NJ,2002. viii+249 pp.

[K-R] Keating, J.P., and Rudnick, Z., The Variance of the number of prime polynomials in short intervals and in residue classes, arXiv:1204.0708v2 [math.NT], 2012 .

[La] Lang, S., Algebraic Groups over Finite Fields, Am. J. Math. Vol. 78, No. 3 (Jul., 1956), pp. 555-563.

Princeton University, Mathematics, Fine Hall, NJ 08544-1000, USA

E-mail address: nmk@math.princeton.edu 